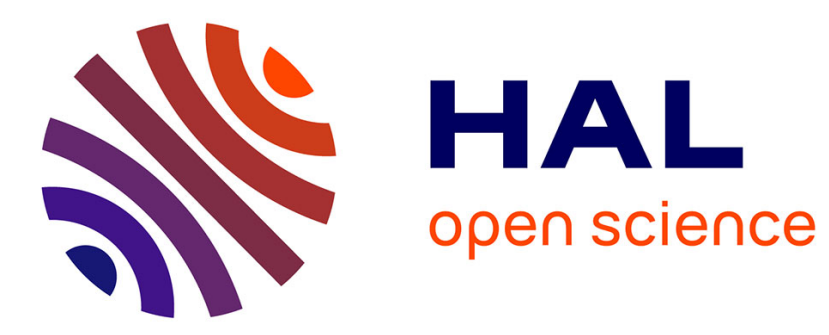

\title{
Synthetic Aperture Interferometric Imaging Using a Passive Microwave Coding Device
}

Ettien Lazare Kpré, Cyril Decroze

\section{To cite this version:}

Ettien Lazare Kpré, Cyril Decroze. Synthetic Aperture Interferometric Imaging Using a Passive Microwave Coding Device. IEEE International Conference on Antennas Measurment and Application (CAMA 2016), Oct 2016, Syracuse, United States. hal-01392889

\section{HAL Id: hal-01392889 \\ https://hal-unilim.archives-ouvertes.fr/hal-01392889}

Submitted on 4 Nov 2016

HAL is a multi-disciplinary open access archive for the deposit and dissemination of scientific research documents, whether they are published or not. The documents may come from teaching and research institutions in France or abroad, or from public or private research centers.
L'archive ouverte pluridisciplinaire $\mathbf{H A L}$, est destinée au dépôt et à la diffusion de documents scientifiques de niveau recherche, publiés ou non, émanant des établissements d'enseignement et de recherche français ou étrangers, des laboratoires publics ou privés. 


\title{
Synthetic Aperture Interferometric Imaging Using a Passive Microwave Coding Device
}

\author{
Ettien Lazare Kpré, Cyril DECROZE \\ XLIM research institute, University of Limoges, \\ 123 avenu Albert Thomas, 87060 LIMOGES CEDEX, France \\ E-mail: ettien.kpre@etu.unilim.fr
}

\begin{abstract}
There has been growing interest in the use of microwave synthetic aperture radiometers since their could solve the trade-off between the image resolution and the antennas aperture size. Compared to classical techniques, SAIR (Synthetic Aperture Interferometric Radiometer) can reach a large field of view (FOV) and high imaging rate. Nevertheless, the architecture of a conventional radiometer still bulky and costly. In this paper, a passive combining technique is proposed to reduce the hardware complexity as well as the dimensional requirements of the receiving antennas. Experimental results are presented to highlight the effectiveness of the proposed technique.
\end{abstract}

\section{INTRODUCTION}

Due to the fact that imaging resolution belongs to the aperture size, real aperture radiometer becomes impracticable for high resolution imaging system. To solve the trade-off between imaging resolution and aperture size, Interferometric aperture Radiometer was suggested in 1980s as an alternative to real aperture radiometer[1]. It can synthesize a large aperture from a few sparsely arranging number of antennas to achieve high spatial resolution without any mechanical scanning.

Since then, numerous systems based on the synthetic interferometric imaging principle have been developed [2]-[3]. Besides, the maturity of microwave technology and imaging algorithms have greatly improved microwave radiometers systems. Nevertheless, high resolution imaging requires a large number of antennas which is directly linked to the number of receivers. In a conventional synthetic aperture radiometer, each receiving element in the physical aperture array is composed of an antenna and a complete receiving chain to ensure the conversion of the RF signal to digital IQ data. As consequence, the complexity of the architecture increases as well as the number of antennas. The contribution of this article is to reduce the number of receivers maintaining the same number of antennas. This is possible using an entirely passive compressive microwave device (i.e an oversized cavity) which can code the $M$ antenna signals to $N$ measured signals $(M \gg N \geq 1)[4]$.

This paper is organized as follows. Section II introduces the interferometry imaging fundamentals. The proposed method is depicted in section III. Experimental measurement results with 4 antennas connected to an oversized cavity is held in section IV to highligh the feasibility of the proposed approach.

\section{INTERFEROMETRY IMAGING FUNDAMENTALS}

The principle of the SAIR is to measure the complex cross-correlation so-called Visibility function $V(u, v)$ of the collected signals $s_{i}(t)$ and $s_{j}(t)$ by respectively the antenna $A_{i}$ and $A_{j}$ [1](Fig 1 ).

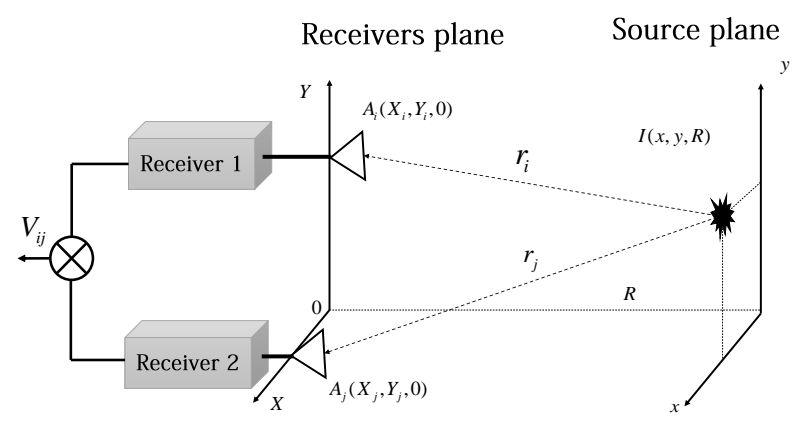

Figure 1. Geometry sketch of a synthetic aperture interferometric radiometer.

$$
V(u, v)=<s_{i}(t) \cdot s_{j}^{*}(t)>
$$

where $\langle.>$ denotes the time integration operator and $(u, v)$ the relative antennas spacing normalized by the wavelength. Based on the Van-Cittert-Zernike theorem [5], the visibility function is related to the brightness temperature by:

$$
\begin{array}{r}
V(u, v) \propto \sum_{\text {source }} \frac{T(x, y) F_{i}(x, y) F_{j}^{*}(x, y)}{r_{i} r_{j}} \\
\times \tilde{r}_{i j}\left(\frac{r_{i}-r_{j}}{c}\right) \cdot e^{-j k\left(r_{i}-r_{j}\right)}
\end{array}
$$

where $T(x, y)$ denotes the normalized brightness temperature of a thermal source located at $(x, y), F_{i}(x, y)$ and $F_{j}(x, y)$ are the normalized radiation pattern of the antennas, $\tilde{r}_{i j}\left(\frac{r_{i}-r_{j}}{c}\right)$ the fringe-wash function which accounts for spatial decorrelation effects. In the limiting narrow-band imaging system, the decorrelation effects are negligible $\left(\tilde{r}_{i j} \approx 1\right)$ [6]. Based on the Eq2, the key factor or the SAIR is the phase due to the path difference $e^{-j k\left(r_{i}-r_{j}\right)}$ between the two antennas. The first order Taylor approximation of the distance $r_{i}$ from the antenna $A_{i}$ to the radiating source is given by : 


$$
\begin{aligned}
r_{i} & =\sqrt{(X i-x)^{2}+\left(Y_{i}-y\right)^{2}+R} \\
& \approx R+\frac{d_{i}^{2}}{2 R}+\frac{x^{2}+y^{2}}{2 R}-\left(\xi X i+\eta Y_{i}\right)
\end{aligned}
$$

where $(\xi, \eta)=(x, y) / R$ are the normalized target coordinates which represent the spatial domain variables, $d_{i}=$ $\sqrt{X_{i}^{2}+Y_{i}^{2}}$ is the distance of the antenna $A_{i}$ to the origin of $(\mathrm{X}, \mathrm{Y})$ axis. $r_{j}$ is deduced with the same approximation, then $k \Delta \tilde{r}$ can be rewritten:

$$
k \Delta \tilde{r}=k\left(r_{i}-r_{j}\right)=\Phi(u, v)-2 \pi(u \xi+v \eta)
$$

Defining the frequency domain variables $(u, v)=\left(X_{i}-\right.$ $\left.X_{j}, Y_{i}-Y_{j}\right) / \lambda . \Phi(u, v)=\pi\left(d_{i}^{2}-d_{j}^{2}\right) / \lambda R$ is the quadratic modified term which can be calculated regardless of the source. Then Eq2 can be rewritten :

$$
\begin{aligned}
V(u, v) & =e^{-i \Phi(u, v)} \times \\
& \iint_{\xi^{2}+\eta^{2} \leq 1} T_{M}(\xi, \eta) e^{i 2 \pi(u \xi+v \eta)} \mathrm{d} \xi \mathrm{d} \eta
\end{aligned}
$$

where $T_{M}(\xi, \eta)$ is the modified brightness temperature given by:

$$
T_{M}(\xi, \eta)=\frac{T(\xi, \eta) F_{i}(\xi, \eta) F_{j}^{*}(\xi, \eta)}{r_{i} r_{j}}
$$

Considering identical radiation pattern for each antenna, the term $F_{i}(\xi, \eta) F_{j}^{*}(\xi, \eta) \approx 1$ and can be ignored, consequently $T_{M}(\xi, \eta) \approx T(\xi, \eta)$. As shown in $\mathrm{Eq} 5$ the visibility function is the product of a quadratic phase and the spatial Fourier transform of the brightness Temperature. Thefore the solving equation of the brightness temperature distribution is given by:

$$
T(\xi, \eta)=\mathscr{F}^{-1}\left[e^{i \Phi(u, v)} V(u, v)\right]
$$

This is the near-field Fourier imaging which can be applied to reconstruct a point-like radiating source. An accurate imaging algorithm can be used for a distributed source imaging [7]-[8]. Nevertheless, high resolution imaging requires a large number of antennas which is directly linked to the number of receivers leading to a cost and complex hardware. This article is devoted to propose a compressive visibility function measurement. This method is cost-efficient since it allows the reduction of the number of receivers compared to that needed in a conventional SAIR. The section bellow shows how to reconstruct the brightness temperature by collecting coded and combined signals.

\section{PASSIVE CODING STRATEGY}

A first passive coding technique was used to perform a digital beam-forming in [9]. This technique was based on the time reversal principle and exploit the frequency diversity of a reverberant cell. It was then extended and applied to SIMO an MIMO imaging [4]-[10]-[11]. Herein, this method is adapted to the SAIR imaging.

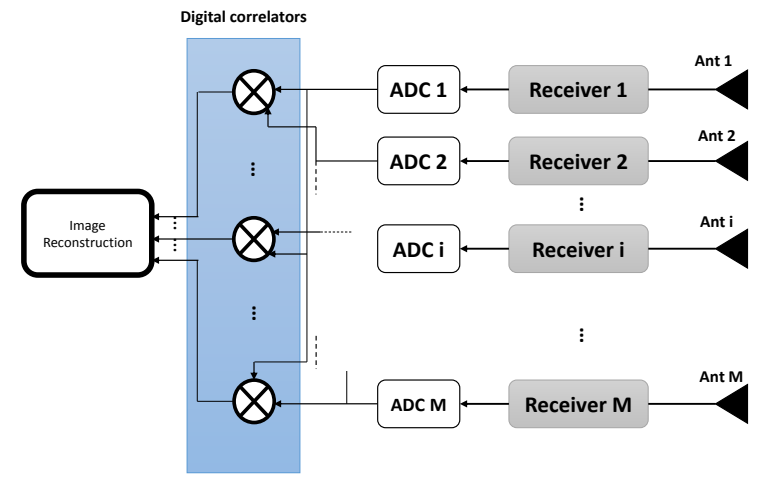

Figure 2. Architecture of a conventional Interferometric radiometer system.

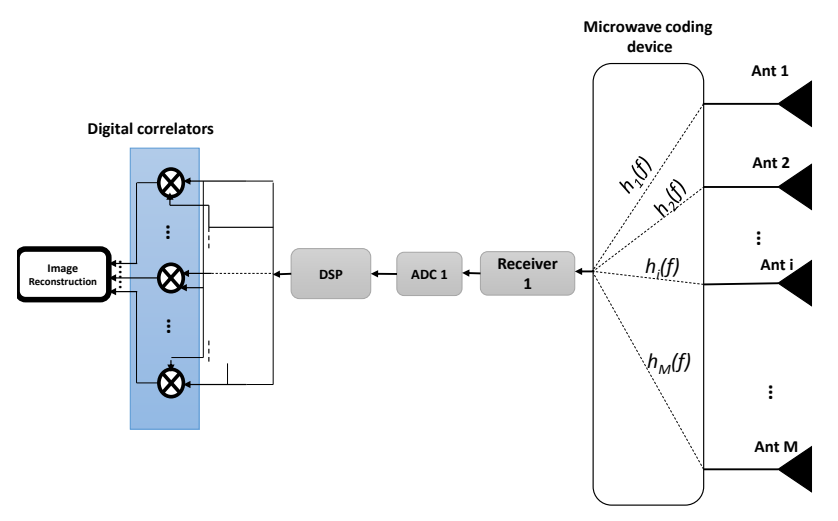

Figure 3. Architecture of an interferometric radiometer with a coding microwave device. $M$ received signals are coded in a single measured signal $c(f)$.

In opposition to a conventional SAIR, the proposed architecture need a single RF chain which significantly reduce the cost of the whole system.

Fig 2 describes a generic setup of the compression technique. The antennas are connected to an entirely passive single output microwave device which codes the antennas signals leading to the number of receiver chains reduction. Then the measured signal $c(f)$ at the device output in frequency domain can be written by Eq.8.

$$
c(f)=\sum_{m=1}^{M} h_{m}(f) s_{m}(f)
$$

where $h_{m}(f)$ corresponds to the device transfer function and $s_{m}(f)$ the received signal by the $m^{t h}$ antenna. For an accurate description of the compressed acquisition, $\mathrm{Eq} 8$ can be reformulated as matrix equation below:

$$
c=\mathbf{h}^{T} \mathbf{s}
$$

$\mathbf{h}$ and $\mathbf{s}$ are $M \times 1$ vectors at a single frequency. Since the principle of the SAIR is to measure the cross-correlation between each pair of the received signals, an estimation of $\mathbf{s}$ 
can be computed for each frequency sample by compensating the impact of the transfer functions $\mathbf{h}$. Due to the minimization of the number of receiver chains, the dimension of the measured signal is significantly less than the received signals. So the linear equation $\mathrm{Eq} 9$ is undetermined. Under this illposed problem, the most efficient method is the regularization to solve it. Tikhonov regularization is an example of suitable regularization method [12] which explicit solution is given by:

$$
\begin{gathered}
\mathbf{h}_{\mu}^{+}=\left(\mathbf{h}^{\dagger} \mathbf{h}+\mu I\right)^{-1} \mathbf{h}^{\dagger} . \\
\mathbf{s}_{r \mu}=\mathbf{h}_{\mu}^{+} c
\end{gathered}
$$

where $\mu$ is the non negative regularization parameter which is used to prevent any ill-conditioning problem. (.) ${ }^{+}$and $(.)^{\dagger}$ are respectively the regularized pseudo-inverse and the transposed conjugate operator. The visibility function can finally be calculated using the conventional formulation :

$$
V_{r}=<s_{r \mu i}(f) \cdot s_{r \mu j}(f)^{*}>
$$

Then the brightness temperature of the target can be computed using Eq.7.

\section{EXPERIMENTAL RESULTS}

Experimental measurement is held to validate the principle of the single port Compressive SAIR (CSAIR). Herein the measurement scenario consists of 4 Vivaldi antennas with an inter-elements spacing $d_{x}=14 \mathrm{~cm}(1.4 \times \lambda)$ in $2.5-3.5 \mathrm{GHz}$ frequency range ( $\lambda$ denotes the central wavelength). A horn antenna is used to model an active noise source. The transmitted signal toward the radiometer is a random Gaussian distribution noise generated by an Agilent M8190A 12GSa/s arbitrary waveform generator.

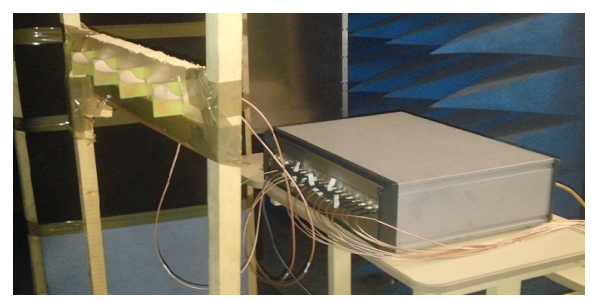

(a)

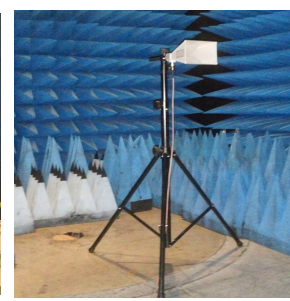

(b)
Figure 4. Conventional SAIR measurement setup. (a) Antennas array connected to the microwave switch and (b) a horn antenna used to model the noise source.

The collected noise signal by each antenna is obtained by the integral of the radiated waves by the horn. The integration time is $\tau=170 \mu \mathrm{s}$. The visibility function is calculated by correlating each pair of received signals. Finally the target brigthness temperature is calculated prior the phased-modified Fourier transform algorithm aforementioned. A conventional SAIR experimental measurement setup is firstly used to reconstruct the horn brightness temperature. A microwave switch (Fig $4 \mathrm{a}$ ) is used to sequentially measure the received signal by each antenna. The signals are recorded using a DSA90404A $20 \mathrm{GSa} / \mathrm{s}$ oscilloscope and jointly post-processed to reconstruct the source brightness temperature.

For the experimental validation of the proposed method, a second SAIR scenario is set up using an empty metallic cavity of outer dimensions of $0.8 \times 0.8 \times 1 \mathrm{~m}^{3}$ as the microwave coding device. Four input of the component are used to connect the antennas as shown in the Fig 5 . The received antenna signals are then coded and combined into a single measured waveform.

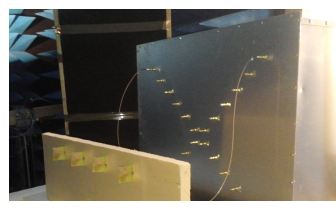

(a)

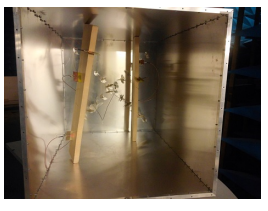

(b)

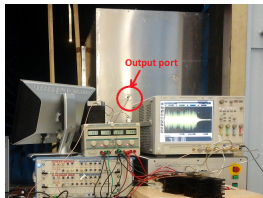

(c)
Figure 5. CSAIR measurement setup with an empty metallic cavity. (a) Antennas array connected to the microwave coding device, (b) Inner view of the cavity and UWB probes randomly placed inside. (c) Backside of the component with a single output port used to collected the coded and combined antenna signals.

Firstly the horn antenna was placed at the quasi-center of the antennas array axis and then shifted along cross-range axis. The imaging distance of the source is $R=3 \mathrm{~m}$.
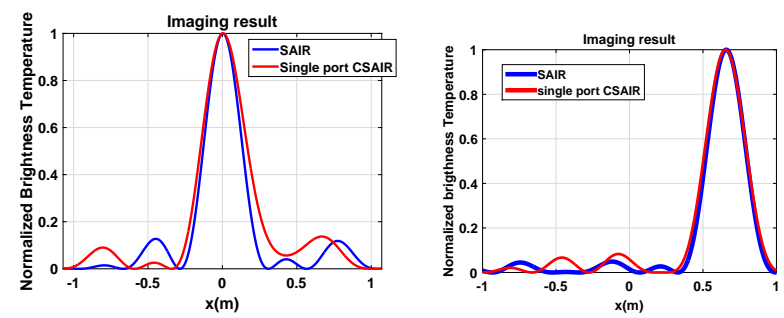

Figure 6. Experimental imaging results of the radiating source. Comparison between conventional SAIR and the proposed method.

The results show that a $1 d$ radiating source image can be reconstructed measuring a single output signal. The resolution is the same for both techniques $(28 \mathrm{~cm})$. The dynamic is about $9 d B$ for the conventional SAIR and $8.63 d B$ for the single port CSAIR. The two imaging techniques results are very similar proving the feasibility of the proposed method.

\section{CONCLUSION}

In this paper, a coding SAIR imaging method have been proposed to reconstruct a radiating source brightness temperature with a single measured signal. In this way, the receiver chains of the conventional SAIR can drastically be reduced using a passive coding device. Herein, an empty metallic cavity have been utilized as the coding device exploiting the frequency diversity. Otherwise the efficiency of the compressed visibility function measurement is coarsely related to the cavity transfer functions correlation level and quality factor. The experiment 
results presented here have been computed considering only four antennas. Future works will focus on enhanced reconstruction methods and imaging algorithms for a high resolution SAIR imaging. A patent is also pending on this new approach.

\section{ACKNOWLEDGMENT}

This work is supported by the French National Research Agency (ANR) within the framework of the funded project "PIXEL". The authors would like to thank all partners of this project.

\section{REFERENCES}

[1] C. S. Ruf, C. T. Swift, A. B. Tanner, and D. M. Le Vine, "Interferometric synthetic aperture microwave radiometry for the remote sensing of the Earth," Geoscience and Remote Sensing, IEEE Transactions on, vol. 26, no. 5, pp. 597-611, 1988.

[2] M. Martin-Neira, Y. Menard, J. M. Goutoule, and U. Kraft, "MIRAS, a two-dimensional aperture synthesis radiometer," in Geoscience and Remote Sensing Symposium, 1994. IGARSS'94. Surface and Atmospheric Remote Sensing: Technologies, Data Analysis and Interpretation., International, 1994, vol. 3, pp. 1323-1325.

[3] D. M. Le Vine, "Synthetic aperture radiometer systems," Microwave Theory and Techniques, IEEE Transactions on, vol. 47, no. 12, pp. 2228-2236, 1999.

[4] T. Fromenteze, E. L. Kpré, D. Carsenat, C. Decroze, and T. Sakamoto, "Single-Shot Compressive Multiple-Inputs Multiple-Outputs Radar Imaging Using a Two-Port Passive Device," IEEE Access, vol. 4, pp. 1050-1060, 2016.

[5] F. Zernike, "The concept of degree of coherence and its application to optical problems," Physica, vol. 5, no. 8, pp. 785 - 795, 1938.

[6] A. Camps, J. Bará, I. C. Sanahuja, and F. Torres, "The processing of hexagonally sampled signals with standard rectangular techniques: Application to 2-D large aperture synthesis interferometric radiometers," Geoscience and Remote Sensing, IEEE Transactions on, vol. 35, no. 1, pp. 183-190, 1997.

[7] J. Chen, Y. Li, J. Wang, Y. Li, and Y. Zhang, "An accurate imaging algorithm for millimeter wave synthetic aperture imaging radiometer in near-field," Progress In Electromagnetics Research, vol. 141, pp. 517-535, 2013.

[8] X. Yao, C. Zheng, J. Zhang, B. Yang, A. Hu, and J. Miao, "Near field image reconstruction algorithm for passive millimeter-wave imager bhu2D-u, Progress In Electromagnetics Research C, vol. 45, pp. 57-72, 2013.

[9] D. carsenat., C. Decroze, "UWB antennas beamform- ing using passive time-reversal device". IEEE Antenna. Wireless Propag. Lett., vol. 11, 2012,779-782.

[10] T. Fromenteze, C. Decroze, D. Carsenat,"UWB passive beamforming for large antenna arrays." IEEE International Conference on Ultra-WideBand, (2014).

[11] T. Fromenteze, E.L. Kpré; C.Decroze, D. Carsenat, O Yurduseven, M.Imani, J. Gollub, D.R. Smith, "Unification of compressed imaging techniques in the microwave range and deconvolution strategy." Proceedings of the 12th European Radar Conference, (2015).

[12] M. Bertero, P. Boccacci,"Introduction to Inverse Problems in Imaging", Institute of physics publishing, 1rst edition 1998. 